\title{
The Action of Thiamine Phosphates on the Contraction of Glycerinated Psoas Muscle
}

\author{
Atsushi Murai and Eisuke Katsura ${ }^{2}$ \\ Nutrition Laboratory, Kyoto University Hospital, Sakyo-ku, Kyoto
}

(Received July 13, 1967)

\begin{abstract}
The action of thiamine and its derivatives on the contraction of glycerinated muscle fiber was investigated.

Neither TMP, TDP, TTP, DCET nor thiamine- $\mathrm{HCl}$ induce the contraction in the absence of ATP.

TDP or TTP, especially TDP, enhance ATP-induced contraction. This enhancing action could not be due to the contaminant calcium but to the change of physicochemical property of contractile protein caused by the binding of thiamine phosphate to it in the same way as ADP.
\end{abstract}

In the previous report it was demonstrated that thiamine and its derivatives in high concentration, especially TPD as well as TTFD, had a remarkable inotropic action on the isolated toad heart (1). This action had been confirmed by other investigators $(2,3)$. However, the mode of this action is as yet unresolved.

On the basis of the present knowledge, actomyosin ATP system (energy utilization), ATP generating system (energy production), plasma membrane, vesicular relaxing factor, etc. are considered to be the site of inotropic action. Thiamine is composed of pyrimidine and thiazole moieties and forms di-, and tri- phosphate of high energy. Therefore, from the similarity as to the chemical structure between thiamine and nucleoside it seems possible that thiamine phosphates interact with actomyosin, altering its physicochemical properties. With respect to this problem no reports have been published except for Greiling's that myosin was able to hydrolyze TTP but not TDP (4). In the present study the action of thiamine and its derivatives on the contraction of glycerol-extracted muscle fiber was examined.

\section{EXPERIMENTAL}

Glycerol-extracted muscle fiber was prepared from psoas muscle of guinea pig according to A. Szent-Gyorgyi's procedure (5). The period of extraction at $-20^{\circ}$ was from 3 days to 5 months. Tension generation was measured isometrically

\footnotetext{
${ }^{1}$ The following abbreviations are used: TPD, thiamine propyl disulfide; TTFD, thiamine tetrahydrofurfuryl disulfide; DCET, dicarboethoxythiamine; TMP, thiamine monophosphate; TDP, thiamine diphosphate; TTP, thiamine triphosphate.

2 村井淳志, 桂 英輔.
} 
with a sensitive force-displacement transducer (displacement of $0.4 \mathrm{~mm}$ corresponded to $20 \mathrm{~g}$ ) and was recorded at intervals of 30 seconds.

The diameter of fiber bundle was approximately $0.2 \mathrm{~mm}$. The medium in which the fiber developed tension had either of the following two compositions; (a) 150 $\mathrm{m} M \mathrm{KCl}, 10 \mathrm{~m} M \mathrm{MgCl}_{2}, 20 \mathrm{~m} M$ Tris-maleate buffer, $\mathrm{pH} 6.8,(b) 90 \mathrm{mM} \mathrm{KCl}, 1$ $\mathrm{m} M \mathrm{MgCl}_{2}, 20 \mathrm{~m} M$ Tris-maleate buffer, $\mathrm{pH}$ 6.8. Final concentrations of ATP were $5 \mathrm{~m} M$ in the medium $(a)$ and $1 \mathrm{~m} M$ in the medium $(b)$. All experiments were carried out at room temperature.

\section{REsUlT}

As is well established, ATP, ADP, CTP, CDP and UTP elicited contraction of glycerinated muscle fiber. ATP and UTP were more active than other nucleotides in this action. However, TMP, TDP, TTP, TPD, DCET or thiamine-HCl were unable to elicit contraction in the absence of ATP. The generated tension reached its maximal level within five minutes after the application of ATP. Five minutes after the application of ATP the above mentioned drugs were added to the medium in which the contracted fiber was immersed. Addition of TMP, TDP or TTP caused further increase of tension. In this case $\mathrm{pH}$ of the medium remained practically unchanged. This action was more pronounced in the case of TDP than in the case of other two drugs (Fig. 1). TDP was effective in a concentration as low as $50 \mu \mathrm{M}$. In the case of thiamine-HCl, TPD and DCET the increased tension after the application of ATP remained unchanged.

In order to pursue a possibility that the action of TDP would be mediated by its inhibitory action on vesicular relaxing factor, glycerol-extracted muscle fiber was treated with $0.015 \%$ benzalkonium chloride solution for three minutes before use (6). However, with this treated fiber the enhancing action of TDP was observed in approximately the same degree.

It is well established that $\mathrm{Ca}^{2+}$ has a pronounced enhancing action on the contraction of glycerinated muscle fiber and the superprecipitation of myosin B. Although Bendall reported that the action of $\mathrm{Ca}^{2+}$ on the contraction of glycerinated muscle fiber reached its maximum in the concentration of $0.2 \mathrm{mM}(7)$, in a calciumdepleted system the action of free $\mathrm{Ca}^{2+}$ can be recognized in the concentration as low as about $0.2 \mu M$ and reaches its maximal level in about $5 \mu M$ concentration $(8,9,10,11)$. In the present study

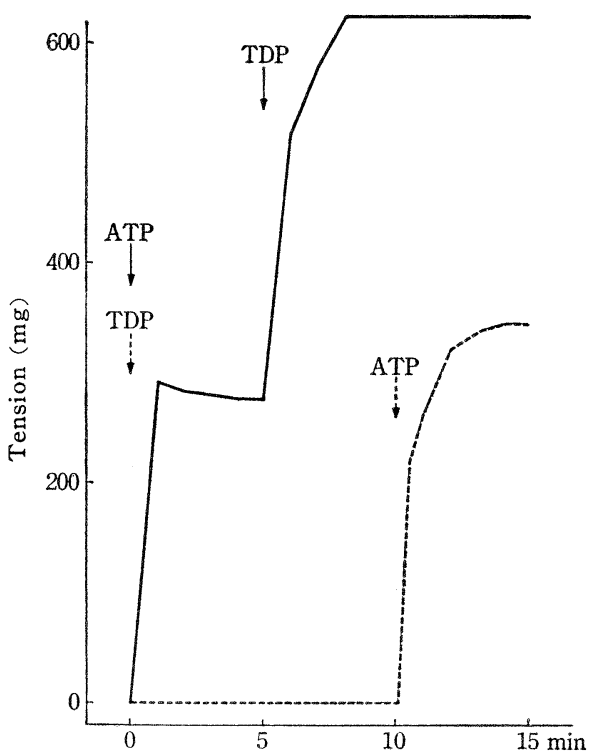

FIG. 1 Action of TDP on Glycerinated Muscle Fiber Before and After Applica. tion of $A T P$

Addition of TDP $(5 \mathrm{mM})$ further increases the tension generated with ATP but cannot elicit contraction in the absence of ATP. Medium (a). 


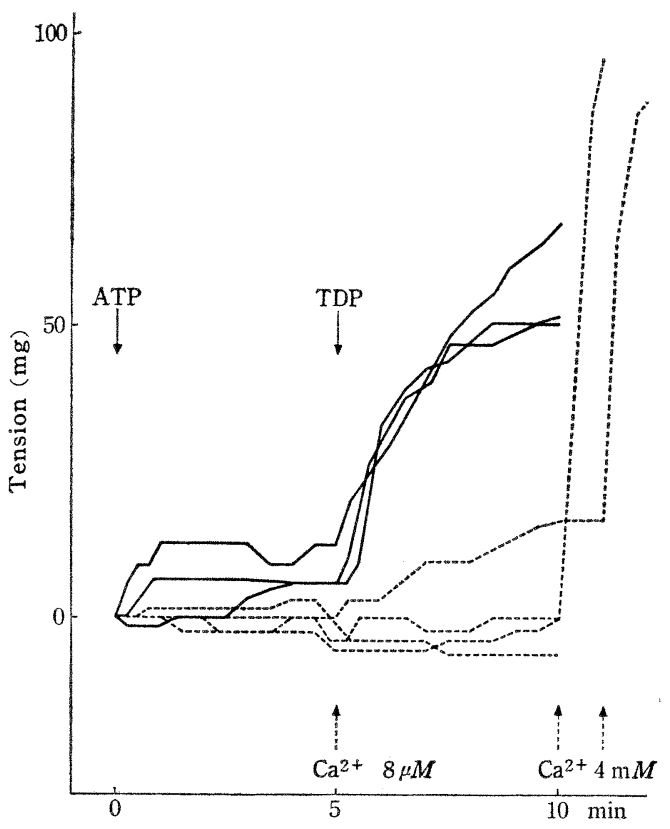

FIG. 2 Action of TDP and $\mathrm{Ca}^{2+}$ on ATP. Induced Contraction

All glycerinated muscle fibers used in this experiment are prepared from a single original muscle bundle. $8 \mu M$ concentration of $\mathrm{Ca}^{2+}$ is equivalent to that derived from contaminant $\mathrm{Ca}^{2+}$ in the $0.5 \mathrm{mM}$ TDP. The period of extraction is three and half months. Medium (b).

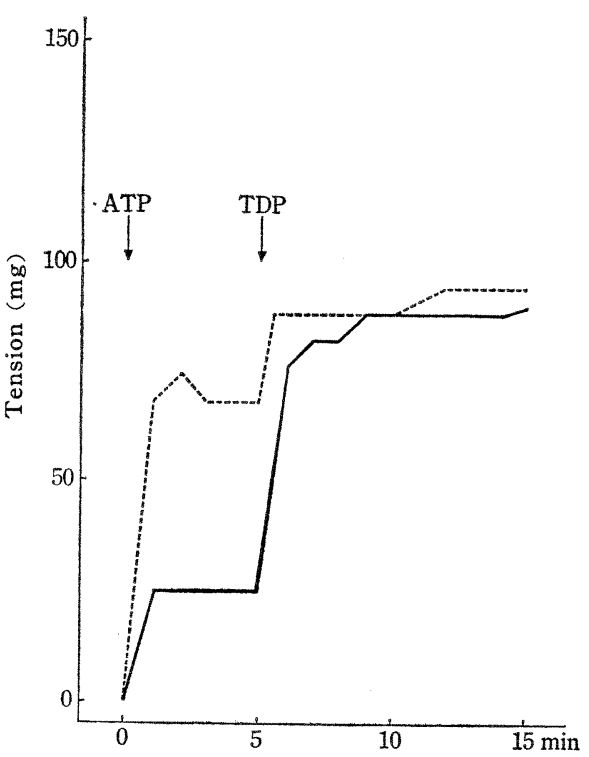

FIG. 3 Action of TDP in Lower Concentration on ATP-Induced Contraction Medium (a). Solid line; $0.5 \mathrm{mM}$ TDP. Broken line; $0.05 m M$ TDP.

$\mathrm{Ca}^{2+}$ enhanced contraction in the concentration above $5 \mu M$ and contaminant calcium in the TDP preparation was flame-photometrically determined to be $0.13 \%$ (molar ratio $=1.6 / 100$ ). On the basis of this result the action of TDP was compared with the action of calcium in concentrations equimolar to the contaminant one. However, the former action was much pronounced than the latter (Fig. 2) and could not be accounted for only by the action of calcium which was contained in the TDP preparation. This result was supported by the fact that TDP was effective in a concentration of $50 \mu M$ and in this case the concentration of calcium was increased only by $0.8 \mu M$ after the addition of TDP (Fig. 3).

\section{Discussion}

In the absence of ATP, neither TMP, TDP nor TTP were able to cause tension generation. Therefore, ATP cannot be substituted with TMP, TDP or TTP in this respect. TDP or TTP, especially TDP, augmented the contraction of glycerinated muscle fiber in the presence of ATP. $\mathrm{Ca}^{2+}$ ion had the same enhancing action which is much more powerful than the action of thiamine phosphates. The drugs used normally contain calcium, as a contaminant, in amounts capable of significantly augmenting the contraction. Consequently, in order to decide 
whether the augmentation of contraction derives from the original action of TDP or not, it is of first necessity to demonstrate that the action thus observed was not due to calcium contamination in the TDP preparation used. With respect to this problem, it is strongly suggested that the contraction of glycerinated muscle fiber is augmented by the original action of TDP from the following three reasons: (a) In the concentration of $50 \mu M$, TDP enhanced ATP-induced contraction. (b) The enhancing action of TDP was observed in the experiment where its enhancing action was compared with the action of equivalent amount of calcium. (c) Furthermore, in the recent experiment on the myosin $\mathrm{B}$ from rabbit skeletal muscle using calcium free TDP as well as TTP treated with Chelex 100, it was demonstrated that TDP as well as TTP enhanced the superprecipitation of myosin B.

With regard to vesicular relaxing factor, benzalkonium chloride treatment did not modify the action of TDP. Consequently, it followed that the action of TDP would not be mediated by vesicular relaxing factor.

What is the mechanism by which TDP augments ATP induced contraction?

(a) Lajtha demonstrated the following facts (12): ADP and creatine phosphate induced contraction of glycerol-extracted muscle fiber and also enhanced ATPinduced contraction of it. On the contrary in the case of actomyosin thread the contraction with ADP occurred only in the presence of myokinase and the contraction with creatine phosphate did only after addition of dialised watery muscle extract. Lajtha suggested that the action of ADP and creatine phosphate depended on ATP synthesis. It is possible that energy rich phosphate of TDP or TTP is transferred to ADP and these thiamine phosphates act as a ATP regenerating system. In fact it is reported that TTP is used as phosphate donor for the phosphorylation of glucose (13). However, if this is the case, it follows that the contraction is rather inhibited than enhanced.

(b) Both TDP and TTP form a complex with $\mathrm{Ca}^{2+}$ or $\mathrm{Mg}^{2+}$. These complex formation results in the decrease of free calcium and magnesium ion concentration. The decrease of free calcium ion concentration brings about an inhibited contraction and the decrease of free magnesium ion concentration has enhancing effect under certain conditions $(14,15)$. If the latter effect overcomes the former, augmented contraction should be observed. However, this explanation is untenable because TDP is effective in a considerably low concentration compared with magnesium concentration present.

(c) The same enhancing action was reported in the case of inorganic phosphate, ADP, creatine phosphate, inosine triphosphate and carnosine $(12,16,17,18,19)$. While ADP competitively inhibits myosin adenosine triphosphatase activity $(20,21,22)$, ADP enhances ATP-induced contraction of glycerinated muscle fiber and ATPinduced flocculation of myofibril. Eisenberg and Moos postulated that the binding of ADP to relaxing site raised the threshold ATP level at which contraction occurred (17). Since the binding of TTP to myosin B was confirmed with lightscattering technique (22), it is possible that this binding is followed by the augmentation of ATP-induced contraction in the same way as ADP. However, the detailed mechanism of this interaction between thiamine phosphate and myosin $B$ remains to be further investigated. Some results with bearing on this problem will be published in the near future. 


\section{REFERENCES}

1. Murai, A., and Katsura, E., Vitamins, 29, 158 (1964), J. Vitaminol., 12, 214 (1964).

2. Tanabe, T., and Kanno, M., Vitamins, 29, 158 (1964).

3. Nakazawa, Y., and Ueno, A., Folia Pharmacol. Japon, 60, 6, $165 \S(1964)$.

4. Greiling, H., and Kiesow, L., Z. Naturforsch, 12b, 672 (1957).

5. Szent-Györgyi, A., Chemistry of Muscular Contraction, Academic Press, 1951, p. 144

6. Ebashi, S., Ebashi, F., and Fujie, Y., J. Biochem, 47, 54 (1960).

7. Bendall, J. R., J. Physiol, 121, 232 (1953).

8. Ebashi, S., J. Biochem, 50, 236 (1961).

9. Weber, A., and Winicur, S., J. Biol. Chem., 236, 3198 (1961).

10. Filo, R. S., and Bohr, D. F., Science, 147, 1581 (1965).

11. Mühlrad, A., and Hegyi, G., Biochim. Biophys. Acta, 105, 341 (1965).

12. Lajtha, A., Enzymologia, 14, 254 (1950).

13. Greiling, H., and Kiesow, L., Z. Naturforsch, 11b, 491 (1956).

14. Mühlrad, A., Kovacs, M., and Hegyi, G., Biochim. Biophys. Acta., 107, 567 (1965).

15. Watanabe, S., Sargeant, T., and Angleton, M., Am. J. Physiol, 207, 800 (1964).

16. Maruyama, K., and Gergely, J., J. Biol. Chem., 237, 1100 (1962).

17. Eisenberg, E., and Moos, C., Arch. Bicohem. Biophys, 110, 568 (1965).

18. Ebashi, S., and Endo, M., Biochemistry of Muscle Contraction, Little, Brown 1964 p. 202.

19. Yun, J., and Parker, C. J. Jr., Biochim. Biophys. Acta., 110, 212 (1965).

20. Kalcker, H. M., J. Biol. Chem, 153, 355 (1944).

21. Blum, J. J., Arch. Biochem. Biophys, 55, 486 (1955).

22. Green, I., and Mommaerts, W. F. H. M., J. Biol. Chem., 210, 695 (1954).

23. Murai, A., and Katsura, E., unpublished data. 